\title{
Acclimating to the Increase in Statin Use in Accountable Care Organizations Based on Changes in Quality Measures: A Report from the Accountable Care Organization Research Network, Services, and Education
}

\author{
Leah Bensimon, PharmD, MSc, and Genevieve Hale, PharmD, BCPS
}

\begin{abstract}
SUMMARY
The Accountable Care Organization Research Network, Services, and Education (ACORN SEED), founded by faculty members at Nova Southeastern University College of Pharmacy, is a group of pharmacists that provides unique pharmacy services to accountable care organizations (ACOs), patient-centered medical homes, and management services organizations to help maximize shared savings and target medication-related issues, while promoting the pharmacy profession and unique learning experiences for pharmacy students within these settings. In this report, ACORN SEED investigators provide a brief overview of the ACO benchmark measures in relation to statin use.

Historically, hyperlipidemia treatment was tailored to meet certain cholesterol levels as a surrogate marker in preventing major adverse cardiovascular events, specifically a low-density lipoprotein cholesterol (LDL-C) level less than $100 \mathrm{mg} / \mathrm{dL}$ as a target goal. In addition, Medicare assessed a health care provider's performance based on this target goal in specific populations (i.e., diabetes and ischemic vascular disease). In 2013, the American College of Cardiology and American Heart Association published updated recommendations removing these LDL-C treatment goals. Rather than treating with cholesterol-lowering medications in an effort to reduce cholesterol levels, patients are now being evaluated for statin therapy use based on 4 benefit groups that focus on the risk of atherosclerotic cardiovascular disease. In 2015, Medicare's shared savings program removed the previous LDL-C goals from its quality measures and now assesses positive performance from the updated guidelines. Currently, under ACO benchmark measure \#42, health care providers are being rewarded for prescribing statin therapy for the prevention and treatment of cardiovascular disease, which reflects updated evidence-based recommendations. With the increase in statin use, randomized controlled trials or ACO validation studies are important in determining future implications on cardiovascular outcomes.
\end{abstract}

J Manag Care Spec Pharm. 2017;23(9):943-44

Copyright $\odot 2017$, Academy of Managed Care Pharmacy. All rights reserved.

T he Accountable Care Organization Research Network, Services, and Education (ACORN SEED), founded by faculty members at Nova Southeastern University College of Pharmacy, is a group of pharmacists that provides unique pharmacy services to accountable care organizations (ACOs), patient-centered medical homes, and management services organizations to help maximize shared savings and target medication-related issues, while promoting the pharmacy profession and unique learning experiences for pharmacy students within these settings. In this report, ACORN SEED investigators provide a brief overview of the ACO benchmark measures in relation to statin use.

\section{ACOs and Quality Benchmarks}

ACOs are groups of doctors, hospitals, and other health care providers that come together voluntarily to give coordinated high-quality care to their Medicare patients. An ACO involves a group of physicians (including primary care) that manages the Medicare beneficiary spending for specific populations under a value-based care model. ACO networks collaborate with other health care providers to optimize patient care and improve health outcomes with reduced costs. ${ }^{1}$ An ACO's integration of health information technology facilitates real-time collaboration among health care providers. Furthermore, an ACO must have a defined process that promotes evidence-based medicine and a patient-centered atmosphere. ${ }^{1}$ Medicare provides a shared savings incentive for ACO networks that meet certain quality measures for specific patient populations (i.e., diabetes, hypertension, ischemic vascular disease, heart failure, and coronary artery disease). ${ }^{2}$ The quality metrics submitted by an ACO are evaluated by the Centers for Medicare \& Medicaid Services (CMS) and the ACO is reimbursed according to its performance on the given benchmarks. These quality measures are evidencebased and can come from updated guidelines or a collaborative decision from the National Committee for Quality Assurance. ${ }^{3}$

\section{Update of Quality Measures Related to Cholesterol}

In 2013, the American College of Cardiology and American Heart Association (ACC/AHA) published an updated recommendation to their guideline for the management of blood cholesterol to remove any low-density lipoprotein cholesterol (LDL-C) target treatment goal less than $100 \mathrm{mg} / \mathrm{dL}^{4}{ }^{4}$ The guideline classifies patients into 1 of 4 statin benefit groups to receive moderate- or high-intensity statin therapy. These 4 statin benefit groups include patients with any form of clinical atherosclerotic cardiovascular disease (ASCVD) consisting of acute coronary syndromes and history of myocardial infarction/transient ischemic attack, stable/unstable angina/stroke, or peripheral arterial disease of atherosclerotic origin; patients with primary LDL-C levels greater than $190 \mathrm{mg} / \mathrm{dL}$; patients with diabetes aged 40-75 years with LDL-C levels between 70 and $189 \mathrm{mg} / \mathrm{dL}$; and patients without diabetes aged 40-75 years with an ASCVD 10 -year risk score greater than or equal to $7.5 \%$. 


\section{Acclimating to the Increase in Statin Use in Accountable Care Organizations Based on Changes in Quality Measures: A Report from the Accountable Care Organization Research Network, Services, and Education}

The ASCVD risk score is derived from the Pooled Cohort Equation (PCE), which incorporates total cholesterol, highdensity lipoprotein, sex, age, race, systolic blood pressure, hypertension treatment, diabetes status, and smoking status. This information estimates a 10 -year risk of a cardiovascular event for a specific patient aged between 20 to 79 years and gives recommendations on the statin intensity needed. ${ }^{4}$ These updated recommendations are based on randomized controlled trials (RCT) that show the benefits of statin therapy in reducing major adverse cardiovascular events (MACE) in high-risk individuals. ${ }^{5}$

The ACC/AHA guideline expert panel, which compiled the most recent blood cholesterol guidelines, has found a lack of RCTs that show treating to LDL-C goals (i.e., less than $100 \mathrm{mg} / \mathrm{dL}$ ) in order to reduce MACE. ${ }^{4}$ Therefore, the recommendation to treat a patient with invalidated surrogate cholesterol measures has been removed from the current guidelines for hyperlipidemia treatment. Patients are now being individualized and treated according to their ASCVD risk in order to reduce MACE. Originally, the ACO quality measure benchmarks \#23 and \#29 targeted LDL-C less than $100 \mathrm{mg} / \mathrm{dL}$ in patients with diabetes or ischemic vascular disease, as well as \#32 that measured drug therapy for lowering LDL-C in patients with coronary artery disease. ${ }^{6}$ As of 2015, CMS has adapted the evidence-based recommendations and removed the quality measures targeting LDL goals and payment policy for ACO networks.

Currently, under ACO quality measure benchmark \#42, health care providers are more broadly being evaluated on the prescription of statin therapy for the prevention and treatment of cardiovascular disease. ${ }^{2}$ With the increase in potential statin users, health care providers are tasked with the decision of whether the statin therapy will clinically benefit all patients that fall under the statin benefit groups. The 2013 guideline does not go through every type of patient and comorbidity, giving health care providers room to make clinical decisions. In addition, statins come with complications that need to be monitored; a patient's hepatic function at baseline and any signs of myalgia need to be addressed. Therefore, the entire clinical picture of a patient needs to be analyzed before blindly following the recommendations in the updated guideline. With that, ACO performance should be properly assessed for quality care rather than for issuance of statin prescriptions.

\section{Future Implications}

Given the increase in statin users since the recommendations were updated in 2013, it is interesting to note that there has been conflicting research regarding the association of the 4 statin benefit groups with positive health outcomes. In an article comparing the PCE ASCVD risk calculator to the European Systematic Coronary Risk Evaluation system (European-SCORE for fatal ASCVD), the PCE ASCVD was found to be superior in estimating cardiovascular outcomes. ${ }^{7}$ However, a recent validation study assessing the PCE ASCVD risk score found that the risk was overestimated and dependent on the contemporary cohort. ${ }^{8}$ The PCE was discordant in predicting a cardiovascular risk by a range of $80 \%-182 \%$.
Therefore, according to the validation study, patients are being overtreated with statins in the long term.

Unfortunately, there is a lack of information on whether aggressive statin treatment based on ASCVD risk will benefit patients' health outcomes. While statin overuse may improve MACE for those patients at risk, not all patients need to be exposed to another medication with potential adverse reactions. Clinicians need to assess a patient's lifestyle along with the patient's medical record before initiating statin treatment. RCTs and ACO network validation studies are needed to assess the effectiveness of the current guidelines in predicting or preventing MACE or death, as well as to evaluate any risk involved with the new statin guidelines. In the meantime, statins will have to do the job.

\section{Authors}

LEAH BENSIMON, PharmD, MSc, and GENEVIEVE HALE, PharmD, BCPS, Nova Southeastern University College of Pharmacy, Palm Beach Gardens, Florida.

AUTHOR CORRESPONDENCE: Genevieve Hale, PharmD, BCPS, Assistant Professor, Nova Southeastern University College of Pharmacy, Palm Beach Gardens Campus, 11501 N. Military Trail, Palm Beach Gardens, FL 33410. Tel.: 561.805.2277;

E-mail: gh341@nova.edu.

\section{DISCLOSURES}

No funding was involved in the preparation of this report. The authors have no conflicts of interest to declare. Both authors contributed equally to data collection, analysis, and manuscript preparation.

\section{REFERENCES}

1. Burke T. Accountable care organizations. Public Health Rep. 2011;126(6):875-78.

2. Centers for Medicare \& Medicaid Services. Medicare program; revisions to payment policies under the Physician Fee Schedule, Clinical Laboratory Fee Schedule, access to identifiable data for the Center for Medicare and Medicaid Innovation Models \& other revisions to Part B for CY 2015. Final rule with comment period. Fed Regist. 2014;79(219):67547-68010.

3. National Committee for Quality Assurance. Accountable care organization accreditation. Available at: http://www.ncqa.org/Programs/Accreditation/ AccountableCareOrganizationACO.aspx. Accessed July 23, 2017.

4. Goff DC Jr, Lloyd-Jones DM, Bennett G, et al. 2013 ACC/AHA guideline on the assessment of cardiovascular risk: a report of the American College of Cardiology/American Heart Association Task Force on Practice Guidelines. Circulation. 2014;129(25 Suppl 2):S49-73.

5. Taylor F, Ward K, Moore TH, et al. Statins for the primary prevention of cardiovascular disease. Cochrane Database Syst Rev. 2011(1):CD004816.

6. Centers for Medicare $\&$ Medicare Services. Table: 33 ACO quality measures. Available at: https://www.cms.gov/Medicare/Medicare-Fee-for-ServicePayment/sharedsavingsprogram/Downloads/ACO-Shared-Savings-ProgramQuality-Measures.pdf. Accessed July 16, 2017.

7. Mortensen MB, Afzal S, Nordestgaard BG, Falk E. Primary prevention with statins: ACC/AHA risk-based approach versus trial-based approaches to guide statin therapy. J Am Coll Cardiol. 2015;66(24):2699-709.

8. Emdin CA, Khera AV, Natarajan P, et al. Evaluation of the Pooled Cohort Equations for prediction of cardiovascular risk in a contemporary prospective cohort. Am J Cardiol. 2017;119(6):881-85. 